\title{
MADERA DE COMPRESIÓN EN Pinus radiata III: PROPIEDADES DE PULPAS KRAFT
}

\section{COMPRESSION WOOD IN Pinus radiata III: KRAFT PULP PROPERTIES}

\author{
J.E. Diaz-vaz ${ }^{1 \oplus}$, R. A. Ananias ${ }^{2}$, L. Valenzuela ${ }^{3}$, M. Torres ${ }^{1}$, S. Rodriguez ${ }^{4}$
}

\section{RESUMEN}

Estudios anteriores en estos mismos árboles de Pinus radiata con fustes levemente inclinados, demostraron que la madera de compresión leve que ellos exhibían presentaba: traqueidas más cortas, mayor densidad, mayor proporción de lignina, mayor proporción de extraíbles en etanol tolueno y menor proporción de alfa celulosa que la madera opuesta y que la madera de árboles sin madera de compresión.

En el presente estudio se analiza las propiedades pulpables de la madera de compresión y la de sus respectivas maderas opuestas de los quince árboles de Pinus radiata estudiados, crecidos en suelos de arena húmeda en Chile. Se estudió separadamente la madera de compresión y la madera opuesta. Con distintas proporciones, de entre 0, 40, 70 y 100 \% de madera de compresión y madera opuesta, se efectuaron pulpajes kraft para cada una de las mezclas.

De estos pulpajes con y sin blanqueo se informan valores de 501 y $695 \mathrm{~kg} / \mathrm{m}^{3}$ de densidad; 28 a $51 \mathrm{Nm} / \mathrm{g}$ en índice de tensión, 18 a $25 \mathrm{mNm}^{2} / \mathrm{g}$ en índice de rasgado, 2.2 a $4.1 \mathrm{kPam}^{2} / \mathrm{g}$ en índice de explosión y rendimiento de 44 a $50.6 \%$ en las mezclas de la pulpa estudiada.

En la pulpa cruda de MC, de la madera pulpable y de la madera exterior aserrable, se registró menor densidad, mayor índice de tensión, de rasgado y de explosión, en comparación con los resultados obtenidos en la pulpa cruda de MO. En el caso de las pulpas blanqueadas, la densidad y el índice de tensión resultaron mayores en la MC.

En el resto de las propiedades las diferencias entre $\mathrm{MC}$ y $\mathrm{MO}$ fueron oscilantes y reducidas. El aumento de la proporción de $\mathrm{MC}$ en la mezcla de $\mathrm{MC}$ y $\mathrm{MO}$, produjo una reducción de rendimiento. Los rendimiento de las mezclas de la madera pulpable indicaron para el $100 \%$ de MO un $50.6 \%$ y para el 100\% de MC un $44 \%$ : En los casos de las pulpas de madera exterior aserrable el 100\% de MO tuvo un rendimiento de de $48.4 \%$ en cambio el $100 \%$ de la MC registró un $44.3 \%$.

Los resultados obtenidos indicaron que la MC leve, que se encuentra en árboles con inclinaciones menores, tuvo un efecto de menor magnitud que él que se produce en la MC severa sobre las características y rendimiento de la pulpa kraft.

Palabras claves: madera de reacción, madera opuesta, pulpa cruda, pulpa blanqueada, rendimiento de pulpa, densidad, índice de tensión, índice de rasgado, índice de explosión.

\footnotetext{
${ }^{1}$ Instituto de Tecnología de Productos Forestales, Universidad Austral de Chile. Valdivia. Chile.

${ }^{2}$ Departamento de Ingeniería en Maderas. Facultad de Ingeniería. Universidad del Bío-Bío. Concepción. Chile. ananias@ubiobio.cl

${ }^{3}$ Facultad de Ciencias Forestales. Universidad de Concepción. Concepción. Chile. lvalenzu@udec.cl

${ }^{4}$ Inger S.A. Concepción. Chile. s_rodriguez@ingerquimica.com

Autor para correspondencia: ^jdiazvaz@uach.cl

Recibido: 15.11.2011 Aceptado: 06.06.2012
} 


\begin{abstract}
Previous studies with radiata pine trees growing slightly leaned towards one side showed that they have small amounts of compression wood with different characteristics than the opposite wood at the other side of the annual rings. These differences include shorter tracheids, higher density, higher proportion of lining, higher proportion of extractives in toluene ethanol, and a smaller amount of alpha-cellulose.

The present study compares the properties of kraft pulp, with and without bleaching, for mixtures of compression and opposite wood obtained from fifteen 22-year-old radiata pine trees that grew in humid sand soils of Chile. The kraft pulp mixture was studied for different proportions of 0,40 , 70 and $100 \%$ compression wood. Considering all mixtures with and without bleaching, the study reports values between 501 and $695 \mathrm{~kg} / \mathrm{m}^{3}$ density, 28 and $51 \mathrm{Nm} / \mathrm{g}$ tension index, 18 and $25 \mathrm{mNm}^{2} / \mathrm{g}$ tear index, 2,2 and 4,1 $\mathrm{kPam}^{2} / \mathrm{g}$ burst index, and $44 \%$ and 50,6\% pulp yield.

It was found that the pulp without bleaching from compression wood (for both the internal pulp and the external lumber areas) had lower density, but higher tension, tear, and burst indexes. The pulp with bleaching from compression wood had both higher density and tension index, but the other properties only show small random differences with respect to opposite wood.

A higher proportion of compression wood in the mixture reduced pulp yield. The yield for the internal pulp area was 50,6 \% with pure opposite wood and $44 \%$ with pure compression wood, while the yield for external lumber area was $48,4 \%$ with pure opposite wood and $44,3 \%$ with pure compression wood. I was concluded, however, that the relatively small amount of compression wood found in these trees had only a minor effect in the properties and yield of kraft pulp.
\end{abstract}

Keywords: Reaction wood, opposite wood, unbleached pulp, bleached pulp, pulp yield, density, tension index, tear index, burst index.

\title{
INTRODUCCIÓN
}

La madera de compresión (MC) tiene características y propiedades diferentes a las que se encuentran en la madera que no es de reacción. La madera de compresión (MC) puede presentar distintos grados de severidad. Correspondiendo a árboles poco inclinados, la denominada MC leve. Este tipo de (MC) presenta características que son más o menos diferentes a la madera de compresión (MC) de grado severo y a la madera de árboles que no tienen MC.

La pérdida momentánea o permanente de la verticalidad de árboles se asocia a cambios de los niveles hormonales en el cambium y a singularidades típicas de la madera que se forma bajo esa circunstancia.

Tanto la MC de coníferas como la madera de tracción en latifoliadas y su disposición en el fuste y ramas laterales tienen que ver con la forma que pueden adquirir los árboles (Tulik y Jura-Morawiec 2011). La madera que se encuentran en el lado opuesto a la zona de reacción, se conoce como madera opuesta (MO).

Muchos de los árboles de plantaciones de Pinus radiata con fustes con pequeñas inclinaciones o curvaturas pueden presentar MC del tipo leve.

Las características más notables que se encuentran en la MC son: mayor proporción de lignina, ausencia de la pared $S_{3}$, traqueidas con secciones redondeadas que presentan a veces intercelulares 
muy notorios, menor largo de traqueidas, mayor ancho de la pared celular de la madera intermedia en el anillo, coloración más oscura y una mayor inclinación de las microfibrillas de la pared secundaria $S_{2}$ que incrementan los cambios dimensionales de dirección axial. Según Donaldson et al. (2012) el conjunto de la pared celular incluyendo la lignificación y la orientación de las microfibrillas pueden variar como resultado de la cantidad y distribución de los polisacáridos no celulósicos.

La MC está asociada a propiedades no deseadas en muchos productos fabricados con este tipo de madera (Timell 1982, Ban et al. 2004). Características anatómicas, físicas y químicas de la MC afectan a productos tales como: pulpa y papel (Timell 1982, Ban et al. 2004), tableros de partículas y tableros de fibras (Roffael et al. 2005, Akbulut y Ayrilmis 2006). La MC ofrece una mayor resistencia a la difusión de la humedad que se explica como resultado de paredes celulares más anchas y ricas en lignina (Tarmian et al. 2012). Por ser una madera con características menos deseadas se ha buscado su empleo en la producción de diferentes materiales incluso compuestos madera-plástico (Buyuksari et al. 2012).

Ensayos realizados anteriormente, con esta misma madera, indicaron diferencias biométricas, físicas y químicas entre MC y MO (Diaz-vaz et al. 2007, 2009). La MC registró, en comparación con la $\mathrm{MO}$, diferencias significativas, de $0.47 \mathrm{~mm}$ de menor largo de traqueidas, una mayor densidad de $33 \mathrm{~kg} / \mathrm{m}^{3}$, lignina en un 1,9\% mayor y un 2,6 \% menor en alfa celulosa. Coarseness y diámetro de traqueidas, por su parte, no presentaron diferencias significativas entre ambas maderas. Todas estas particularidades presentes en esta MC de grado leve, suponen algún efecto más o menos importante en las propiedades de pulpa kraft en que ella participa.

Las particularidades de esta MC leve se consideró que podrían afectar en mayor o menor grado las características de las pulpas, de allí que el objetivo de este estudio se centró en contrastar las propiedades de las pulpas de la MC leve con las de la MO producidas con madera de árboles de Pinus radiata con inclinaciones menores de sus fustes.

\section{MATERIALES Y MÉTODOS}

Para este estudio se dispuso de la madera de quince árboles de Pinus radiata pertenecientes a un rodal de 22 años, creciendo en un sitio de arena ( $\left.37^{\circ} 20^{\prime} S-71^{\circ} 30^{\prime} \mathrm{W}\right)$ en Chile. La madera de estos árboles se empleó en estudios anteriores relacionados con particularidades anatómicas, física y químicas de la MC (Diaz-vaz et al. 2007, 2009).

Los árboles muestreados tenían fustes rectos con inclinaciones no mayores a $10^{\circ}$ respecto a la vertical. Rodelas de $5 \mathrm{~cm}$ de espesor correspondientes a trozos pulpables y aserrables fueron utilizadas para los ensayos del presente estudio.

De los quince árboles disponibles, cinco árboles con las mayores dimensiones del sitio, proporcionaron la MC y la MO considerada como madera exterior de trozos aserrables. De los otros diez árboles se obtuvo la MC y la MO correspondiente a la madera de trozos considerados pulpables.

Sobre las superficies pulidas de las rodelas se identificó la MC por la excentricidad de la médula, el ancho de los anillos, la coloración rojiza y la apariencia similar a madera tardía que presentó la madera intermedia de los anillos.

Las zonas de $\mathrm{MC}$ y de $\mathrm{MO}$, con edades del cambium comparables de cada rodela, separadamente fueron astilladas manualmente. De las zonas definidas como MC y como MO que tenían la altura de $25 \mathrm{~mm}$ de la rodela, se astillaron manualmente a un espesor de $5 \mathrm{~mm}$ y con anchos entre 15 y $25 \mathrm{~mm}$. Estas astillas fueron clasificadas en un harnero plano con perforaciones circulares seleccionándose 
los tamaños entre 1 1/8 a 3/8 de pulgada.

Los pulpajes se realizaron en un digestor de marca MK System de 6,5 litros de capacidad equipada con bomba de recirculación y calefactor eléctrico.

Las condiciones para los pulpajes fueron: peso de madera seca, $800 \mathrm{~g}$; álcali activo $22 \% \mathrm{bms}$. (como óxido de sodio); sulfidez $27 \%$; temperatura máxima $175^{\circ} \mathrm{C}$; tiempo hasta $100{ }^{\circ} \mathrm{C}$ alcanzados a los 60 min y tiempo entre $100^{\circ} \mathrm{C}$ y $175^{\circ} \mathrm{C}$ en 90 min; tiempo a temperatura máxima a $175^{\circ} \mathrm{C}$ durante $40 \mathrm{~min}$. La relación licor - madera fue de de 4/1.

Las condiciones empleadas permitieron obtener pulpas con índice kappa entre 30 y 34 . Los resultados de los pulpajes correspondieron a: índice kappa, TAPPI T $236 \mathrm{~cm}-85$; álcali residual, TAPPI T $625 \mathrm{~cm}-85$; sólidos totales, TAPPI T 650 om- 89.

Las pulpas obtenidas, sin refinar y refinadas se evaluaron desde el punto de vista de sus propiedades físico-mecánicas. Las determinaciones se ciñeron a los siguientes procedimientos: refinación PFI, TAPPI T 248 sp-00; formación de hoja, TAPPI T 205 sp-95; resistencia al drenaje método Schopper-Riegler ( ${ }^{\circ}$ SR), ISO 5267-1:1979; propiedades físicas, TAPPI T 220 sp-95.

Con la MC y la MO recolectada de todas las rodelas se dispuso de madera para realizar una serie de mezclas de MC/MO con: 0\%, 40 \% 70 \% y $100 \%$ de MC. Se analizó separadamente la madera pulpable de la madera exterior aserrable. Se aseguró que la MC tuviera igual edad del cambium que la MO y que participaran en igual proporción en el caso de cada una de las mezclas (Diaz-vaz et al. 2007). Con estas pulpas se realizaron los respectivos análisis de propiedades mencionados en el párrafo anterior.

Se efectuaron cuatro blanqueos, dos para la madera pulpable y dos para la madera exterior aserrable. En cada caso se consideró la secuencia Cd-Eop-D-E-D, blanqueo estándar de laboratorio, aplicado en ese momento en la industria, partiendo con pasta cruda de kappa entre 30 y 34 aproximadamente. Este blanqueo estándar se aplicó en función del contenido de lignina de la pulpa de modo de afectar lo menos posible la resistencia de la pulpa. La tabla 1 resume las condiciones de cada una de las etapas de blanqueo empleadas.

Tabla 1. Secuencia del blanqueo aplicado a las pulpas.

\begin{tabular}{|c|c|c|c|c|c|}
\hline $\begin{array}{c}\text { Etapas } \\
\text { del blanqueo }\end{array}$ & $\begin{array}{c}\text { Cd } \\
\text { (70 \% de cloro } \\
\text { y 30 dicloro) }\end{array}$ & $\begin{array}{c}\text { Eop } \\
\text { (Extracción } \\
\text { alcalina) }\end{array}$ & $\begin{array}{c}\mathrm{D} \\
\text { (Dióxido } \\
\text { agregado) }\end{array}$ & $\begin{array}{c}\mathrm{E} \\
\text { (Extracción } \\
\text { alcalina) }\end{array}$ & $\begin{array}{c}\mathrm{D} \\
\text { (Dióxido } \\
\text { agregado) }\end{array}$ \\
\hline Carga $(\%)$ & 8,07 & 2,00 & 1,81 & 2.00 & 0,20 \\
\hline Consistencia $(\%)$ & 3 & 10 & 10 & 10 & 10 \\
\hline Temperatura $\left({ }^{\circ} \mathrm{C}\right)$ & 18 & 80 & 60 & 80 & 75 \\
\hline Tiempo $(\mathrm{min})$ & 30 & 60 & 30 & 60 & 180 \\
\hline
\end{tabular}

\section{RESULTADOS}

La influencia sobre el rendimiento que provocó la $\mathrm{MC}$ en las pulpas de madera exterior aserrable y en la madera pulpable, se resume en el tabla 2. La que muestra los cambios de rendimiento al aumentar el porcentaje de $\mathrm{MC}$ en la mezcla MC/MO. Con índice kappa de 30 a 34 el rendimiento alcanzado en los casos de las pulpas de madera pulpable y de madera exterior aserrable, se redujeron 
al aumentar la proporción de MC en las mezclas de: 0\%, 40\%, 70\% y 100\% de MC en la mezcla MC/ MO.

Tabla 2. Rendimiento de la pulpa en las mezcla de madera de compresión y de madera opuesta para pulpables y exterior/aserrables.

\begin{tabular}{|c|c|c|c|}
\hline $\begin{array}{c}\text { Mezcla } \\
\text { compresión/opuesta }\end{array}$ & $\begin{array}{c}\text { Densidad de la madera } \\
\left(\mathrm{kg} / \mathrm{m}^{3}\right)\end{array}$ & $\begin{array}{c}\text { Rendimiento clasificado } \\
(\%)\end{array}$ & No KAPPA \\
\hline \multicolumn{4}{|c|}{ PULPABLE } \\
\hline $0 / 100$ & 337 & 50,6 & 31 \\
\hline $40 / 60$ & 361 & 48,2 & 34 \\
\hline $70 / 30$ & 365 & 46,5 & 31 \\
\hline $100 / 0$ & 378 & 44,0 & 30 \\
\hline \multicolumn{5}{|c|}{ EXTERIOR ASERRABLE } \\
\hline $0 / 100$ & 371 & 48,4 & 34 \\
\hline $40 / 60$ & 380 & 46,9 & 31 \\
\hline $70 / 30$ & 392 & 45,4 & 30 \\
\hline $100 / 0$ & 414 & 44,3 & \\
\hline
\end{tabular}

En las mezclas MC/MO la MC participó con madera de una densidad de $378 \mathrm{~kg} / \mathrm{m}^{3}$ y la de la pulpa de la MO con madera de una densidad de $337 \mathrm{~kg} / \mathrm{m}^{3}$, esto son valores de la madera de las trozas pulpables (Tabla 2). La densidad de la madera exterior aserrable fue de $414 \mathrm{~kg} / \mathrm{m}^{3}$ en el caso de la MC y de $371 \mathrm{~kg} / \mathrm{m}^{3}$ en la MO. Tanto en la madera pulpable como en la exterior aserrable el rendimiento clasificado de las mezclas disminuyó a medida que aumentaba la proporción de MC (Tabla 2)

En la tabla 3 se aprecia que la densidad de las pulpas crudas resultaron menor que las correspondientes blanqueadas. Por el contrario los índices de tensión, de rasgado y de explosión, resultaron mayores en las pulpas sin blanqueo.

La madera pulpable presentó mayor densidad que las respectivas de la madera exterior aserrable. Esto tanto en las pulpas de MC como en las de MO. Lo mismo se registró en las pulpas crudas y las pulpas blanqueadas. Resultado similar se puede apreciar en el índice de tensión e índice de explosión. Diferente resultó el índice de rasgado de la pulpa cruda y blanqueada de la madera pulpable que presentó menor valor que la de la madera exterior aserrable (Tabla 3).

Tabla 3. Propiedades físicas de la pulpa cruda y la pulpa blanqueada, sin refinar, de la madera de compresión y opuesta de madera pulpable y madera exterior aserrable.

\begin{tabular}{|l|c|c|c|c|c|}
\hline & $\begin{array}{c}\text { Resistencia } \\
\text { al drenaje } \\
\left({ }^{\circ} \mathrm{SR}\right)\end{array}$ & $\begin{array}{c}\text { Densidad } \\
\left(\mathrm{kg} / \mathrm{m}^{3}\right)\end{array}$ & $\begin{array}{c}\text { Tensión } \\
(\mathrm{Nm} / \mathrm{g})\end{array}$ & $\begin{array}{c}\text { Rasgado } \\
\left(\mathrm{mNm}^{2} / \mathrm{g}\right)\end{array}$ & $\begin{array}{c}\text { Explosión } \\
\left(\mathrm{kPam}^{2} / \mathrm{g}\right)\end{array}$ \\
\hline Pulpable cruda opuesta & 15 & 561 & 516 & 246 & 4,0 \\
\hline Pulpable cruda compresión & 14 & 626 & 485 & 220 & 4,1 \\
\hline Pulpable blanqueada opuesta & 14 & 632 & 299 & 183 & 2,4 \\
\hline Pulpable blanqueada compresión & 14 & 695 & 369 & 203 & 3,1 \\
\hline Exterior aserrable cruda opuesta & 14 & 508 & 439 & 255 & 3,6 \\
\hline Exterior aserrable cruda compresión & 15 & 501 & 449 & 242 & 4,0 \\
\hline Exterior aserrable blanqueada opuesta & 13 & 625 & 287 & 253 & 2,2 \\
\hline Exterior aserrable blanqueada compresión & 13 & 638 & 299 & 201 & 2,4 \\
\hline
\end{tabular}


Un resumen de las propiedades de la pulpas de la madera pulpable y exterior aserrable, tanto blanqueadas como crudas de la MO y MC, se presentan en la tabla 4 todas ellas a un drenaje de $\left.18^{\circ} \mathrm{SR}\right)$. En general la densidad y el índice de tensión resultaron con valores más altos en la MC que en la MO. Solo un caso resultó diferente y fue el de la madera aserrable que el caso de la pulpa cruda la MO resultó de un valor mayor que la MC. Para el índice de explosión y el de rasgado los valores resultaron mayores en la pulpa de la MO. Tampoco varían estos resultados con valores de drenaje constante (tabla 4).

Tabla 4. Propiedades físicas de las pulpas a drenaje constante de 18 (oSR).

\begin{tabular}{|c|c|c|c|c|c|c|c|c|}
\cline { 2 - 9 } \multicolumn{1}{c|}{} & \multicolumn{4}{c|}{ Madera pulpable } & \multicolumn{5}{c|}{ Madera aserrable } \\
\cline { 2 - 10 } \multicolumn{1}{c|}{} & Pulpa cruda & \multicolumn{2}{c|}{ Pulpa blanqueada } & \multicolumn{2}{c|}{ Pulpa cruda } & \multicolumn{2}{c|}{ Pulpa blanqueada } \\
\cline { 2 - 9 } \multicolumn{1}{c|}{} & MC & MO & MC & MO & MC & MO & MC & MO \\
\hline $\begin{array}{c}\text { Densidad } \\
\left(\mathrm{kg} / \mathrm{m}^{3}\right)\end{array}$ & 859 & 831 & 899 & 851 & 837 & 820 & 892 & 859 \\
\hline $\begin{array}{c}\text { Explosión } \\
(\mathrm{kPam} / \mathrm{g})\end{array}$ & 7,2 & 7,3 & 6,3 & 6,6 & 6,6 & 7,3 & 7,2 & 7,3 \\
\hline $\begin{array}{c}\text { Rasgado } \\
(\mathrm{mNm} / \mathrm{g})\end{array}$ & 10,729 & 12,491 & 10,742 & 11,579 & 14,038 & 17,285 & 12,876 & 15,675 \\
\hline $\begin{array}{c}\text { Tensión } \\
(\mathrm{Nm} / \mathrm{g})\end{array}$ & 102,825 & 96,292 & 89,956 & 81,194 & 85,955 & 93,095 & 89,722 & 87,105 \\
\hline
\end{tabular}

La pulpa de MC y también la de MO fueron afectadas por el blanqueo. Se registró un incremento en la densidad, tanto en la pulpa de madera pulpable como en las de la madera exterior aserrable. Por su parte, los índices de: tensión, rasgado y explosión, en la mayoría de los casos, presentaron valores menores en la pulpa blanqueada que los registrados en la pulpa cruda (tabla 3). Tampoco variaron los resultados con valores de drenaje constante (tabla 4)

La refinación incidió en las propiedades de la pulpa de $\mathrm{MC}$ y de $\mathrm{MO}$, cruda y blanqueada, aumentando sus valores. A mayor refinación PFI, los valores del drenaje ( $\left.{ }^{\circ} \mathrm{SR}\right)$, como era de esperar, aumentaron. Especialmente notorio fue el aumento del drenaje en la pulpas blanqueadas de la MO y la $\mathrm{MC}$ en la madera pulpable y aserrable valores que sobre pasaron notoriamente los alcanzados por las pulpas crudas (Figura 1).

Los cambios que se originan con variaciones del drenaje se ilustran, para las propiedades físicas en la figura 1. 


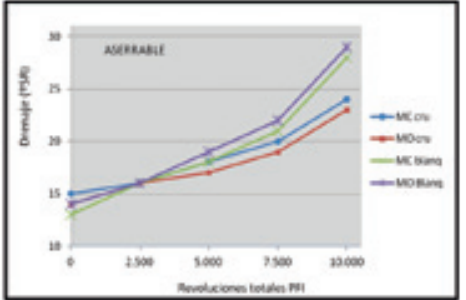

(a)Aserrables

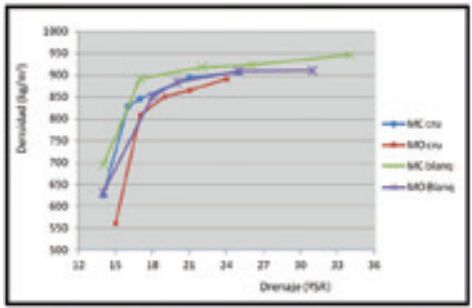

(c)Pulpables

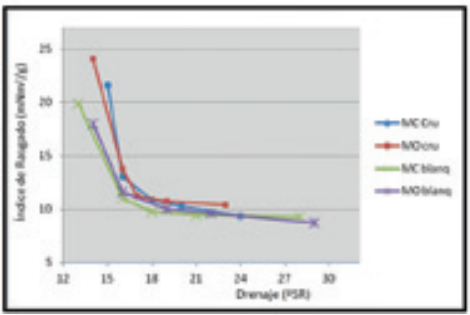

(e)Pulpables

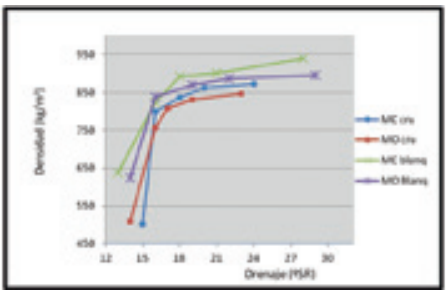

(g)Aserrables

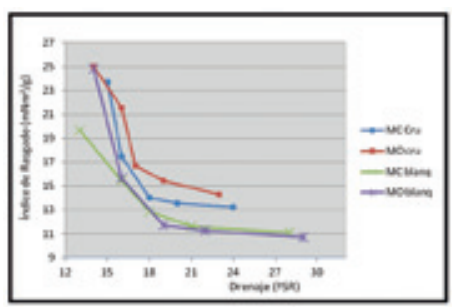

(i)Aserrables

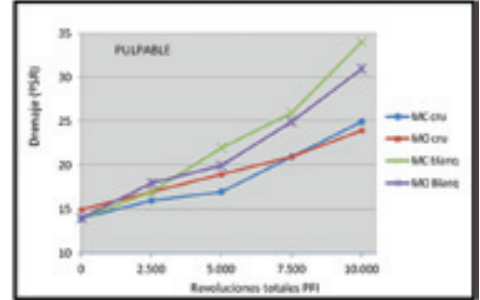

(b)Pulpables

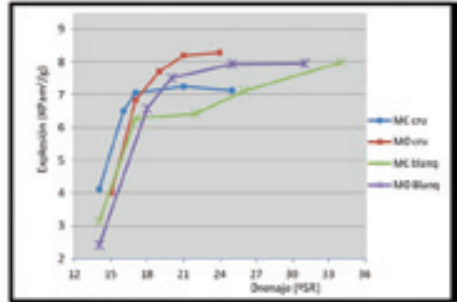

(d)Pulpables

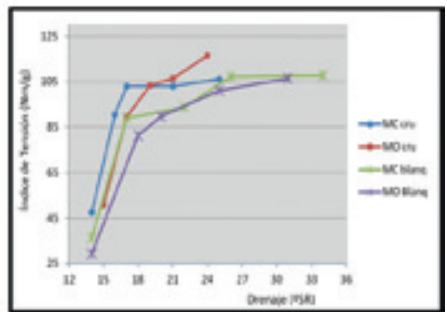

(f)Pulpables

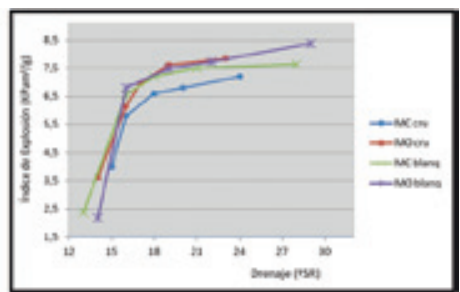

(h)Aserrables

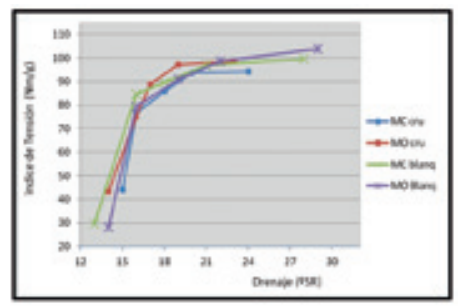

(j)Aserrables

Figura 1. Resistencia al drenaje, densidad, índice de explosión, índice de rasgado e índice de tensión en pulpas crudas y blanqueadas en madera de compresión y opuesta, de trozos pulpables y de trozos aserrables (exterior aserrables).

MO cru=CRUDA, OPUESTA; MC cru=CRUDA, COMPRESIÓN; MO blanq=BLANQUEADA, OPUESTA; MC blanq=BLANQUEADA, COMPRESIÓN);

Drainage resistance, Density, Tensión index, Tear index, and Burst index, to unbleached and bleached compression and oposite pulp, from slabwood and pulp wood.

MO cru= unbleached, opposite pulp; MC cru= unbleached, compression pulp; MO blanq=bleached, opposite pulp); MC blanq=bleached, compression pulps). 
La pulpa blanqueada de la madera pulpable, tanto MO como MC permitieron mayor drenaje que la pulpa cruda, sin que ello significara un detrimento importante de los diversos índices. Sin embargo, el índice de rasgado de la $\mathrm{MC}$ y $\mathrm{MO}$, tanto de la madera pulpable como de la aserrable, mostraron reducciones fuerte con el aumento del drenaje. Redujeron sus disminuciones en valores cercanos a 19 a 20 ( $\left.{ }^{\circ} \mathrm{SR}\right)$. Las pulpas blanqueadas, por su parte, continuaron la disminución del rasgado con valores más o menos estables hasta drenajes algo mayores a $33\left({ }^{\circ} \mathrm{SR}\right)$ en las pulpables y con cerca de los 28 ( $\left.{ }^{\circ} \mathrm{SR}\right)$ en aserrables (Figura 1). La densidad de la madera pulpable en el caso de la MC blanqueada aumentó sobrepasando los 27 ( $\left.{ }^{\circ} \mathrm{SR}\right)$. Otros índices para las pulpas, incluso mejoraron tal como sucedió con el índice de explosión, algo más notorio en el caso de la pulpa blanqueada que mejoró por sobre los 34 ( $\left.{ }^{\circ} \mathrm{SR}\right)$ de drenaje resultando algo similar con el índice de tensión (Figura 1).

\section{DISCUSIÓN}

La madera de $P$. radiata, principal especie usada en la fabricación de pulpa y papel en Chile, requiere para maximizar las operaciones de producción: el mejor entendimiento de las singularidades físicas y químicas en los componentes de la madera y como ellas se relacionan con las características finales de ese y otros productos.

De allí que la presencia de MC, con sus particularidades químicas, biométricas y físico-mecánicas, pueden influir en productos obtenidos a partir de esta madera.

Ensayos realizados anteriormente, con la madera de los mismos árboles de Pinus radiata utilizados en este ensayo, indicaron diferencias biométricas, físicas y químicas entre MC y MO (Diaz-vaz et al. 2007, 2009). La MC registró, en comparación con la MO, diferencias significativas, de $0,47 \mathrm{~mm}$ de menor largo de traqueidas, una mayor densidad de $33 \mathrm{~kg} / \mathrm{m}^{3}$, lignina en un $1,9 \%$ mayor y un 2,6 $\%$ menor en alfa celulosa. Coarseness y diámetro de traqueidas, por su parte, no presentaron diferencias significativas entre ambas maderas. Estas singularidades en esta MC de grado leve, permitieron presuponen algún efecto más o menos importante en las propiedades de las pulpas kraft en que ella participen.

El menor rendimiento obtenido en los pulpajes de MC se puede relacionar con la mayor proporción de lignina que presenta esta madera (Einspahr et al.1969, Uprichard 2002, Ban et al. 2004). En el presente estudio al aumentar la proporción de MC en la mezcla de MC-MO, se produjo, como era esperable, una reducción del rendimiento con una baja de 50,6 \% a $44 \%$, en el caso de la madera pulpable y de $48,4 \%$ a $44,3 \%$ en la madera exterior aserrable. (Tabla 2).

La reducida diferencia de rendimiento entre la MC y la MO puede explicarse considerando que la madera utilizada mostró, en el estudio anterior, diferencias de solo 1,9\% en la proporción de lignina y de 2,6 \% en la proporción de alfa celulosa entre MO y MC de grado leve. Lo mismo en los casos de la madera pulpable y exterior aserrable, diferencias que también fueron pequeñas (Diaz-vaz et al. 2009).

Lo anterior explicaría lo reducido de las diferencias en el rendimiento registrado entre la MC y la MO, y también la diferencia entre MC de pulpable y MC de madera exterior aserrable, que es semejante a las diferencias informadas por otros autores (Uprichard y Gray 1973, Ban et al. 2004, Mancosky et al. 2005) pero sin embargo contrastan con otros informes en los que probablemente estos dos tipos de madera pueden tener mayores diferencias en densidad y largo de traqueidas (Lohrasebi $e t$ al. 1999, Ban et al. 2004, Torres et al. 2005, Mancosky et al. 2005)

La MO pulpable presentó un rendimiento más alto que la MO de la madera exterior aserrable de 50,6 y $48,4 \%$ respectivamente (Tabla 2). Diferencias coincidentes con los valores de 47,8 y $46,8 \%$ 
según Uprichard y Gray (1973) para madera de Pinus radiata.

A pesar de las diferencias en las propiedades de las pulpas de trozas pulpables y aserrables con presencia de MC de grado leve, el rendimiento de esta madera no puede considerarse que sea afectado de manera importante por la presencia de esta MC de grado leve.

La MC y la MO disponible para los ensayos, que se obtuvo de las rodelas de todos los árboles, conformó una cantidad de madera reducida que permitió realizar un pulpaje con la madera de todos los árboles para cada uno de los ensayos, sin posibilidad de realizar repeticiones. La reducida cantidad de madera comparable, entre MC y MO especialmente en cuanto a edades fisiológicas similares, no permitió realizar pulpaje para cada árbol. De allí que las diferencias son solo valores promedio del conjunto de las pulpas conseguidas con el total de los árboles. Como no se realizó análisis estadístico, no se puede asegurar que las diferencias sean significativas. Sin embargo, al comparar los resultados obtenidos con resultados de otros autores pareciera que los valores no necesariamente son equivocados.

La resistencia al drenaje de las pulpas, (Figura 1) mostraron diferencias reducidas entre la MC y $\mathrm{MO}$ en todo el rango de refinación estudiado. Las pulpas blanqueadas tampoco resultaron muy diferentes entre la $\mathrm{MO}$ y la MC, pero los drenajes fueron mayores en los casos de las pulpas blanqueadas tanto en las pulpas de madera pulpable como en las de madera exterior aserrable.

Las pulpas blanqueadas de la madera pulpable tanto en la MO como en la MC permitieron mayores drenajes que las pulpas crudas. Esto es válido en los casos de la densidad de la pulpa, el índice de explosión, el índice de rasgado y de tensión. Comportamiento similar se obtuvo en las pulpas de madera aserrable.

Las pulpas de MC y MO de la madera pulpable y exterior aserrable, mostraron un comportamiento diferente con la refinación. La pulpa de la madera pulpable obtuvo valores mayores de drenaje que la pulpa de la madera de exterior aserrable, esta diferencia se hizo más notoria al aumentar el grado de refinación (Figura 1).

La MC con traqueidas de paredes más anchas y diámetros de lúmenes menores a los de la MO, supone un efecto sobre el refinado que estaría relacionado con la mayor dificultad al colapso de esas fibras y por ello sería esperable menor drenaje en las pulpas de MC. Los resultados obtenidos aquí indican sin embargo, un comportamiento errático en ambas pulpas. Este resultado variable puede deberse, en parte a que en esta misma muestra de madera, en un ensayo anterior, se determinó que no existían diferencias significativas en el diámetro de fibras de la madera tardía de MC y la de MO. (Diaz-vaz et al. 2007). Resultado coincidente con lo informado por Donaldson et al. (2004) en Pinus radiata.

Por otra parte, el menor largo de traqueidas determinado en esta MC (Diaz-vaz et al. 2007) debería producir mayores valores de resistencia al drenaje en la MC. A todo lo anterior podría agregarse singularidades anatómicas de la sub-estructura de la pared celular, presente en las traqueidas de la MC, que pudieran incidir también en el drenaje resultante con la refinación.

En distintos procesos de pulpaje, de diferentes especies, se ha informado que las pulpas de MC mostraron distintas calidades a las obtenidas con $\mathrm{MO}$ y a la de madera de árboles que no presentaban MC (Lohrasebi et al. 1999, Torres et al. 2005, Mancosky et al. 2005). La mayor diferencia química que presenta la MC, en comparación con la MO y la de árboles sin MC, es su mayor proporción de lignina y su menor proporción de alfa celulosa. A estas desigualdades se agregan diferencias anatómicas, incidiendo todas ellas en los diferentes resultados que se obtienen entre las pulpas de MC, MO y la de la madera de árboles sin MC. 
Los valores de densidad se incrementaron rápidamente con el aumento del drenaje alcanzando valores que tienden a estabilizarse cercanos a los 18 ( $\left.{ }^{\circ} \mathrm{SR}\right)$. La MC presentó valores algo mayores que la $\mathrm{MO}$ en algunos niveles de refinación. Según lo informado por Einspahr et al. (1969) quienes consideran, en los casos de madera tardía y de MC, que las fibras de paredes celulares anchas de la MC dificultan el colapso y por ello producen pulpa con densidad aparente reducida. Sin embargo, por el contrario, diferencias de densidad entre MC y MO también pueden deberse al menor largo de fibras de la MC, singularidad que puede asociarse a pulpas con mayor densidad. También es necesario considerar como probable efecto en esta misma dirección que influyan: la ausencia de la $\mathrm{S}_{3}$, la presencia de fracturas helicoidales en la $S_{2}$ y anomalías de la $S_{1}$ presentes en la MC (Uprichard y Gray 1973).

El índice de tensión de la madera pulpable obtenido, es resultado del diámetro del lumen y ancho de la pared celular en este tipo de madera. Ellos inciden en la resistencia al colapso de las fibras y en los enlaces que se producen entre ellas. Ello es válido tanto en casos de la MC y la MO. Resultado coincidente con lo informado para Pinus radiata (Uprichard y Gray 1973, Burdon et al. 1999, Kibblewhite 1980, Uprichard 2002).

El índice de tensión, como la mayoría de los índices de las pulpas de MC y MO, aumentó muy notoriamente con la refinación a 2500 revoluciones, Este resultado se muestra con un drenaje que se incrementa entre drenajes de 16 a 18 ( $\left.{ }^{\circ} \mathrm{SR}\right)$. El índice de tensión de la pulpa de MC resultó muy similar al de la MO, especialmente en las pulpas aserrables. Se podría entender el menor índice de tensión de la pulpa de MC obtenido, considerando: la mayor densidad de la MC, las paredes más anchas y la mayor dificultad para el colapso que tienen las traqueidas de la MC. Esto explicaría también el menor índice de tensión en las pulpas de madera exterior aserrable (Figura 1j). La MC con menores posibilidades de enlaces entre fibras produciría un menor índice de tensión. De igual manera incrementos de la densidad de la madera en arboles jóvenes de Pinus taeda se asociaron con reducción del índice de tensión de sus pulpas. El índice de tensión está relacionado con el porcentaje de madera tardía y con el ancho de la pared celular (Einspahr et al. 1969). Las pulpas blanqueadas permiten mayor drenaje por el efecto del blanqueo sobre la densidad de la pulpa y las modificaciones que produce en la pared celular.

La pulpa de la madera de mayor edad del cambium, madera aserrable, registró valores más altos de índice de rasgado que la madera más joven, resultado que coincide con lo informado por distintos autores. Este resultado se puede explicar si se considera que las fibras largas de la madera madura dan pulpas con mejores propiedades de rasgado (Einspahr et al. 1969, Kibblewhite 1973, 1980, Melo et al. 1988, Drost y Shewchunk 2003).

El índice de rasgado de madera aserrable, obtenido en la pulpa de $\mathrm{MC}$, se redujo más con el aumento del drenaje hasta los 24 ( $\left.{ }^{\circ} \mathrm{SR}\right)$, valores que se reducen menos en el caso de la MO, esto en las pulpas crudas ya que las pulpas blanqueadas reducen levemente sus valores hasta 28 ( $\left.{ }^{\circ} \mathrm{SR}\right)$. Este comportamiento es bastante similar en la madera pulpable (Figura 1i). Este resultado puede asociase con mayor densidad y fibras de paredes más anchas de la MC, que colapsan con más dificultad que las fibras de la MO. El blanqueo, en las pulpas aserrables mostraron una reducción que permitió mayores drenajes que la que se produjo en la madera pulpable. El efecto de la mayor densidad de la madera aserrable, puede explicar este resultado (Einspahr et al.1969). Así es como se puede esperar una menor reducción del índice de rasgado con mayores valores de drenaje. También se debe considerar, para los valores de rasgado de la MC, las fibras más cortas que posee la MC en ambos tipos de maderas pulpables y aserrable.

En cuanto al comportamiento del índice de explosión registrado en la pulpa de la madera exterior con el aumento del drenaje, este se relacionaría con la menor densidad de esta pulpa que se obtiene de madera con mayor densidad, la que así se relaciona con el menor índice de explosión (Einspahr et al.1969, Kibblewhite 1980, Melo et al. 1988, Torres et al. 2005). 
La pulpa de MC presentó valores de explosión menos afectados con el drenaje que los registrados en la pulpa de la MO (Figura 1d). Esto se puede explicar por las fibras de paredes más anchas, con un menor colapso, a lo que se suma las células más cortas de la MC, que disminuyen la posibilidad de enlaces entre fibras y producen una menor densidad aparente de la pulpa.

El efecto del blanqueo permitió tener valores de explosión menos afectados por el drenaje, especialmente en el caso de la MO de la madera aserrable. En el caso de la pulpa de madera pulpable la pulpa blanqueada de MC fue en aumento a medida que aumentaba el drenaje.

La menor densidad aparente de pulpa se relaciona con madera de mayor densidad y así con pulpas de menor índice de explosión (Einspahr et al. 1969, Uprichard y Gray 1973, Kibblewhite 1980, Torres et al. 2005). Esto explicaría los resultados obtenidos en el índice de explosión en el caso de la madera de exterior aserrable. La mayor edad de esta madera, madera más densa, influiría en el menor índice de explosión de su pulpa.

Diferencias en los resultados obtenidos entre las pulpas de madera pulpable y de madera exterior aserrable se presentaron en: densidad, índice de tensión, rasgado y explosión. Las pulpas de la madera pulpable, resultaron con mayor densidad, mayor índice de tensión y explosión. Por el contrario, el índice de rasgado fue menor en la madera pulpable. Estos resultados pueden explicarse por diferencias de edad (del cambium) entre estos dos tipos de madera.

La mayor densidad de la pulpa de la madera pulpable se puede explicar por la menor densidad y menor largo de las fibras que presentaba esta madera (Diaz-vaz et al. 2007), en comparación con la madera correspondiente a los anillos más alejados de la médula.

El efecto del blanqueo significó que la pulpa presentara una mayor resistencia al drenaje que la pulpa cruda, diferencia que se incrementó con la refinación (Figura 1).

La densidad de la pulpa blanqueada, en ambos tipos de madera, pulpable y exterior aserrable, resultó mayor que la cruda, diferencia que se redujo con la refinación (Figura 1). Como el blanqueo puede producir un ablandamiento de la pared celular, esto supone una mayor compactación y por ello un aumento de la densidad (Torres et al. 2005). En cuanto a la pulpa cruda el refinado de la pulpa cruda promueve el colapso de las fibras y con ello el incremento de la densidad. Este resultado es concordante con lo informado para MC de Pinus radiata (Uprichard y Gray 1973) que indica, para igual refinación, diferencias de densidad de pulpa de madera juvenil, de madera madura y de MC, pero que con un batido de 15000 revoluciones los resultados cambian y las densidades resultan similares.

Las diferencias de índice de tensión en las pulpas blanqueadas y en las crudas de MC y MO se redujeron con la refinación. En cuanto al índice de rasgado, las pulpas blanqueadas registraron valores menores y similares a las crudas especialmente con el refinado. Anteriormente se indicó que el blanqueo incrementaba la densidad de las pulpas y esta mayor densidad aparente de las pulpas se asocia a rasgados menores (Einspahr et al. 1969). Así podría explicarse los valores menores de rasgado que se pueden observar también en las pulpas blanqueadas (Figura 1).

Los resultados obtenidos indican que con la refinación aplicada, las pulpas crudas incrementaron su índice de explosión en más de un 200\%. Por su parte, las pulpas blanqueadas, incrementaron su índice de explosión en más de un 300 \%. Este resultado es atendible si se considera que el blanqueo produce cambios en la composición química y ablandamiento en la pared celular lo que origina una mayor compactación, una menor densidad aparente y un mayor índice de explosión (Torres et al. 2005). 


\section{CONCLUSIONES}

La pulpa de MC leve de árboles con inclinaciones reducidas, presentó propiedades y rendimientos no siempre diferentes a los que se obtuvo en la pulpa de MO de esos mismos árboles.

La refinación modificó todas las variables en los primeras 2500 revoluciones y su efecto resultó variable según la propiedad analizada. La resistencia al drenaje varió con el refinado según se tratara de pulpa cruda, de pulpa blanqueada, de la madera pulpable o de la madera de exterior aserrable.

En la pulpa cruda de MC, de la madera pulpable y de la madera exterior aserrable, se registró menor densidad, mayor índice de tensión, de rasgado y de explosión, en comparación con los resultados obtenidos en la pulpa cruda de MO. En el caso de las pulpas blanqueadas, la densidad y el índice de tensión resultaron mayores en la MC. En el resto de las propiedades la diferencia entre MC y MO fue oscilante.

El aumento de la proporción de $\mathrm{MC}$ en la mezcla de $\mathrm{MC}$ y $\mathrm{MO}$, produjo una reducción del rendimiento. Los rendimiento de $\mathrm{MC}$ y de $\mathrm{MO}$ fueron 50,6 \% y $44 \%$, en los casos de la madera pulpable y de $48,4 \%$ en MC y de $44,3 \%$ en MO, en madera exterior aserrable.

Los resultados obtenidos indican que la MC leve, que se encuentra en árboles con inclinaciones menores, tiene un efecto de menor magnitud que el que produce la MC severa, sobre las propiedades físicas y el rendimiento de la pulpa kraft.

La MC leve que se encuentra en árboles con inclinaciones menores, origina modificaciones menores en las propiedades y rendimiento de la pulpa kraft en estos árboles de Pinus radiata.

\section{AGRADECIMIENTOS}

Los autores agradecen al Comité de Investigaciones Básicas de CMPC Celulosa, Laja, Chile, por su apoyo financiero.

\section{REFERENCIAS}

Akbulut, T.; Ayrilmis, N. 2006. Effect of compression wood on surface roughness and surface absorption of medium density fiberboard. Silva Fennica 40(1):161-167.

Ban, W.; Mancosky, D.; Lucia, L. 2004. Evaluation of the pulping response of juvenile and mature black spruce compression wood. Cellulose Chemistry and Technology 38(1-2):79-85.

Burdon, R.; Kibblewhite, R.; Riddell, M. 1999. Wood density and kraft fibre and pulp properties of four Pinus radiata provenances. New Zealand Journal of Forestry Science 29(2):214-224.

Buyuksari, U.; Ayrilmis, N.; Akbulut,T. 2012. Compression Woods as a Reinforcing Fikkeer for Thermoplastic Composites. Journal of applied polymer science 123(3): 1740-1745.

Diaz-vaz, J.E.; Fernandez, A.; Valenzuela, L.; Torres, M. 2007. Madera de compresión en Pinus radiata D.Don: I Características anatómicas. Maderas. Ciencia y tecnología 9(1):29-43.

Diaz-vaz, J.E.; Ananías, R.; Rodriguez, S.; Torres, M.; Fernandez, A.; Poblete, H. 2009. Compression wood in Pinus radiata II: Density and chemical composition. Maderas. Ciencia y tecnología 11(2):139-151. 
Donaldson, L.; Grace, J.; Downes, G. 2004. Within-tree variation in anatomical properties of compression wood in radiata pine. IAWA Journal 25(3):253-271.

Donaldson, L.; Lloyd, A.; Konx, J. 2012. Localization on Cell Wall Polysaccharides in Normal and Compression Wood of Radiata Pine: Relationships with Lignification and Microfibril Orientation. Plant Physiology 158(2):642-653.

Drost, C.; Ni, Y.; Shewchunk, D. 2003. Effect on mature and juvenile wood from five wood species on kraft pulp strength. Pulp and Paper-Canada 104(11):33-36.

Einspahr, D.; van Buijtenen, J.; Peckham, J. 1969. Pulpping characteristics of ten-year loblolly pine selected for extreme wood specific gravity. Silvae Genetica 18(3):57-61.

Kibblewhite, R. 1973. Effect of beating and wood quality on radiata pine kraft paper properties. New Zealand Journal of Forestry Science 3(2):220-239.

Kibblewhite, R. 1980. Radiata pine corewood and slabwood, and their interrelations with pulpo and handsheet. New Zealand Journal of Forestry Science 10(3):533-550.

Lohrasebi, H.; Mabee, W.; Roy, D. 1999. Chemistry and pulping feasibility of compression wood in black spruce. Journal of Wood Chemistry and Technology 19(1-2):13-25.

Mancosky, D.; Ban, W.; Lucia, L. 2005. Chemical study of the variation in the bleaching and pulping response of predominantly juvenile and mature. Northern Black Spruce Fractions. Ind, Eng. Chem. Res. 44(6):1652-1659

Melo, R.; Paz, J.; Carrasco, V.; Bello, N. 1988. Interrelación entre las propiedades de una celulosa kraft y la materia prima usada para su fabricación. Celulosa y Papel 3 (4):15-20.

Roffael, E.; Essiamah, S.; Diaz-vaz, J.E.; Schneider, T.; Dix, B. 2005. Untersuchungen über den Einfluß von Reaktionsholz (Druckholz) und Normalholz der Fichte auf die Eigenschaften von mitteldichten Faserplatten (MDF). Forstarchiv 76 (6):206-214.

Tarmian, A.; Remond, R.; Dashti, H.; Perre, P. 2012. Moisture diffusion coefficient of reaction woods; compression wood of Picea abies L. and tension wood of Fagus sylvatica 1. Wood Science Technol. 46(1-3):405-417.

Timell, T. 1982. Recent progress in the chemistry and topochemistry of compression wood. Wood Sci. Technol.16:83-122.

Torres, L.; Melo, R.; Colodette, J. 2005. Pulpa kraft blanqueada a partir de Pinus tecunumanii. Bosque 26(2):115-122.

Tulik, M.; Jura-Morawiec, J. 2011. Reaction wood and the crown architecture. Sylwan 155(12): 808-815.

Uprichard, J. 2002. Pulp and paper from radiata pine. Appita and Forest Research. 478 p.

Uprichard, J.; Gray, J. 1973. Papermaking properties of kraft pulps from New Zealand grown softwoods. Appita 27(3):185-191. 
